\title{
LABORATORY METHODS
}

\section{Generation of rodent and human osteoblasts}

\author{
Sarah EB Taylor ${ }^{1}$, Mittal Shah ${ }^{2,3}$ and Isabel R Orriss ${ }^{3}$ \\ ${ }^{1}$ Department of Orthopaedic Surgery, Stanford University School of Medicine, Stanford, CA, USA. ${ }^{2}$ Department of Surgery \\ and Cancer, Imperial College London, London, UK. ${ }^{3}$ Department of Comparative Biomedical Sciences, Royal Veterinary \\ College, London, UK.
}

\begin{abstract}
This paper describes the isolation, culture and staining of primary osteoblasts from neonatal rodents and human samples. The calvaria and long-bone assays allow direct measurement of bone matrix deposition and mineralisation, as well as producing osteoblasts at defined stages of differentiation for molecular and histological analysis. Culture of human osteoblasts enables cell function to be investigated in targeted patient groups. The described methods will provide a step-by-step guide of what to expect at each stage of the culture and highlight the varied tissue culture conditions required to successfully grow osteoblasts from different sources. A special focus of this paper is the methods used for analysis of bone mineralisation and how to ensure that nonspecific mineral deposition or staining is not quantified.
\end{abstract}

BoneKEy Reports 3, Article number: 585 (2014) | doi:10.1038/bonekey.2014.80

\section{Introduction}

Osteoblasts, the bone-forming cells, are derived from mesenchymal stem cells. In vitro culture of osteoblasts constitutes a central part of research into the regulation of bone formation. Many approaches have been developed to study osteoblasts including primary cell cultures, bone organ cultures and immortalised osteoblast-like cell lines (for example, MC3T3-E1, MG-63 and SaOS-2). Together, these techniques have provided abundant information about the processes regulating osteoblast proliferation, differentiation, survival and function.

Several techniques for obtaining primary osteoblasts have now been described. ${ }^{1-3}$ These methods use cells isolated from different skeletal locations (for example, long bones, calvaria) and animal models (for example, human, rat and mouse). The in vitro culture of calvarial osteoblasts from neonatal rats is an important and widely used technique for studying the regulation of osteoblast function. This method provides high numbers of cells and is relatively easy to do, making it ideal for experimental purposes. Osteoblasts isolated from long bones are less frequently used but also represent an important source of cells. ${ }^{4}$ Although the mechanisms of bone formation differ between the calvaria (intramembranous ossification) and the long bones (endochondral ossification) recent work has shown that osteoblasts isolated from these different anatomical locations behave in a similar manner in vitro. ${ }^{4}$

The calvarial and long-bone osteoblast bone-formation assays have several advantages, that make them good models for studying the cellular responses to exogenous stimuli. First, they allow the key function of osteoblasts, namely bone formation, to be quantified. ${ }^{1}$ Second, it enables the processes of bone matrix deposition and mineralisation to be studied independently. ${ }^{5}$ Finally, osteoblasts can be studied at clearly identified stages of differentiation from immature, proliferating cells through to the mature bone-forming osteoblasts.

These methods also allow osteoblast activity to be studied in an environment that is relatively free from the influence of other cell types normally found in bone such as endothelial and haematopoietic cells. Furthermore, it allows the extracellular environment (for example $\mathrm{pH}$ and $\mathrm{pO}_{2}$ ) to be tightly controlled and if required, manipulated, in a manner which is not possible in vivo or using bone organ cultures. ${ }^{6,7}$

Human osteoblasts, from several anatomical sources, have also been widely used to study osteoblast physiology. ${ }^{2,8-11}$ Human cells obtained from explant bone tissues consist of a heterogeneous cell population that predominantly consists of early osteoblasts. In vitro these cells can be differentiated into mature, bone-forming osteoblasts by the addition of glucocorticoids, vitamin $\mathrm{D}$ and bone morphogenetic proteins. ${ }^{8-11}$ Although slower growing compared with neonatal rodent cells, the use of human osteoblasts allows cell function to be investigated in patients suffering from age-related bone diseases.

Here we describe methods for the isolation of rat calvarial and long-bone osteoblasts from the same animal. We also describe how these methods should be adapted to successfully culture mouse osteoblasts, which appear to require more nutritional support in vitro. Finally, we describe how to culture primary human osteoblasts from bone samples.

Correspondence: Dr IR Orriss, Department of Comparative Biomedical Sciences, Royal Veterinary College, London NW1 OTU, UK.

E-mail: iorriss@rvc.ac.uk 


\section{Materials and Methods}

\section{Materials}

All solutions, instruments and tissue culture plastics must be sterile. Tissue culture reagents were obtained from Life Technologies (Paisley, UK) and, unless stated, all other reagents from Sigma-Aldrich (Poole, UK).

- Animals: Cells are obtained from the calvaria and long bones of neonatal rats ( $2-3$ days) and mice (2-5 days). One neonatal rat will produce $7 \times 10^{6}-10^{7}$ and $5 \times 10^{6}$ cells from the calvaria and long bones, respectively. One mouse calvaria will typically yield $3-5 \times 10^{6}$ cells.

- Human specimens: Cells are obtained from the trabecular region of the humeral head from patients undergoing total shoulder replacements. The amount of trabecular specimen received will vary depending on surgeries, but a specimen of $\sim 5 \mathrm{~mm}^{3}$ will produce $1-2 \times 10^{6}$ cells. Other anatomical sources of bone can also be used.

- Phosphate-buffered saline (PBS): For storing tissues and washing cells. The addition of $100 \mathrm{Uperml}$ penicillin, $100 \mu \mathrm{g}$ per $\mathrm{ml}$ streptomycin and $0.25 \mu \mathrm{g}$ per $\mathrm{ml}$ amphotericin (known as antibiotic/antimitotic or $\mathrm{AB} / \mathrm{AM}$ ) is recommended for human cells (PBS $+\mathrm{AB} / \mathrm{AM})$.

- Alpha-modified essential medium ( $\alpha M E M)$ : Add 10\% foetal calf serum, $2 \mathrm{~mm}$ L-glutamine and AB/AM. Rat and human cells can also be cultured in Dulbecco's modified Essential medium (DMEM), supplemented as $\alpha$ MEM (Note 1). This supplemented medium is usually stable for $\sim 4$ weeks.

- Osteogenesis aMEM (osMEM) or DMEM (osDMEM): To $\alpha M E M$ or DMEM add $50 \mu \mathrm{g}$ per $\mathrm{ml}$ ascorbate, $2 \mathrm{~mm} \beta$-glycerophosphate (Note 2) and $10 \mathrm{~nm}$ dexamethasone (not mouse cells, Note 3). Always make fresh on day of use.

- Sodium hydroxide $(\mathrm{NaOH}): 6 \mathrm{~m} \mathrm{NaOH}$ to alter the $\mathrm{pH}$ of the culture medium if required (Note 4).

- Trypsin-EDTA: 0.25\% (w/v) trypsin with $1 \mathrm{~mm}$ EDTA.

- Accutase: with PBS containing $0.5 \mathrm{~mm}$ EDTA and $3 \mathrm{mg}$ per I phenol red for human cell disassociation (Note 5).

- Collagenase: $0.2 \%(w / v)$ collagenase solution (Type II collagenase from Clostridium histolyticum, made up in Hank's balanced salt solution).

- Fixative: $2.5 \%$ glutaraldehyde in $\mathrm{dH}_{2} \mathrm{O}, 70 \%$ ethanol or $4 \%$ paraformaldehyde in PBS (pH 7.4).

- Alizarin red stain for mineralised bone nodules: $1 \%(w / v)$ alizarin red in $\mathrm{dH}_{2} \mathrm{O} \mathrm{pH} 5.5$.

- Alkaline phosphatase (tissue nonspecific alkaline phosphatase (TNAP)) staining: Required components - naphthol ASMX-phosphate, dimethylformamide and fast blue $b$ salt.

- TNAP activity: Sensolyte pNPP TNAP assay kit (Anaspec, Fremont, CA, USA).

- Sirius red stain for deposited collagen: Sircol dye reagent (Biocolor, County Antrim, UK).

- Masson's trichrome collagen staining: required components-Bouin's fixative, Weigert's haematoxylin solution, $0.5 \%$ acid fushcin solution with $5 \%$ acetic acid, phosphomolybdic acid solution, $2 \%$ methyl blue solution with $2.5 \%$ acetic acid and 1\% acetic acid solution.

- Tissue culture plastics: Large petri dishes $(100 \mathrm{~mm}), 5 \mathrm{ml}$ flatbottomed tubes, $15 \mathrm{ml}$ and $50 \mathrm{ml}$ centrifuge tubes, 25 and $75 \mathrm{~cm}^{2}$ tissue culture flasks and 6-, 12- or 24-well tissue culture plates (Note 6).
- Dissection tools: Scalpels and blades (no.20), rongeurs, bone cutters, tweezers and scissors.

\section{Methods}

Isolation of primary osteoblasts from neonatal rodent calvaria

- Euthanise 2-6 neonatal rats (2-3 days) or mice (2-5 days) and sterilise with $70 \%$ ethanol. Remove the head using large scissors; retain the body for long-bone isolation.

- Remove the skin and brain tissue from the skull using a scalpel and tweezers.

- Cut away the jaw and carefully scrape off any excess tissue and cartilage from around the calvaria.

- Cut the calvaria in half and place in a flat-bottomed 5-ml tube; wash with PBS.

- Repeat steps 2-5 as required.

- Incubate in $0.25 \%$ trypsin $(500 \mu \mathrm{l}$ per calvaria) for $10 \mathrm{~min}$ at $37^{\circ} \mathrm{C}$. Remove and discard the trypsin solution; wash in aMEM or DMEM.

- Incubate in $0.2 \%$ collagenase solution $(600 \mu$ l per calvaria) for $30 \mathrm{~min}$ at $37^{\circ} \mathrm{C}$.

- Remove the collagenase digest, discard and replace with fresh solution for $60 \mathrm{~min}$ at $37^{\circ} \mathrm{C}$.

- Transfer the final digest to a 15-ml centrifuge tube. Wash any remaining calvaria with $\alpha$ MEM or DMEM $(5 \mathrm{ml})$ and add the solution to the final digest.

- Spin at $1500 \mathrm{~g}$ for $5 \mathrm{~min}$. Discard the supernatant and resuspend the cells in $\alpha$ MEM or DMEM ( $1 \mathrm{ml}$ per calvaria).

- Rat cells: Add $20 \mathrm{ml}$ of $\alpha$ MEM or DMEM and $1 \mathrm{ml}$ cell suspension per $75 \mathrm{~cm}^{2}$ flask (1 calvaria per flask).

- Mouse cells: Pool the cell suspensions; add $17 \mathrm{ml}$ of $\alpha \mathrm{MEM}$ and $3 \mathrm{ml}$ cell suspension per $75 \mathrm{~cm}^{2}$ flask ( 3 calvaria per flask). If the calvaria need to be kept separate (for example, different genotypes) expand cells in a $25 \mathrm{~cm}^{2}$ flask ( $7 \mathrm{ml}$ of $\alpha$ MEM plus $1 \mathrm{ml}$ cell suspension).

- Incubate the flask at $37{ }^{\circ} \mathrm{C} / 5 \% \mathrm{CO}_{2}$ until the cells become confluent ( $\sim 3$ days and 5-6 days for rat and mouse cells, respectively).

Isolation of primary osteoblasts from the long bones of neonatal rats

- Remove the limbs from the body by cutting with sharp scissors taking care to preserve as much of the limb as possible.

- Using a scalpel cut off the paws and cut the limb in half (at the joint).

- Remove the skin and scrape away the soft tissue. Cut off the epiphyses and place the bone fragments into a flat-bottomed $5 \mathrm{ml}$ tube. Wash and vortex in PBS to remove any residual soft tissue.

- Incubate the bone fragments in trypsin (1 ml per animal) for $10 \mathrm{~min}$ at $37^{\circ} \mathrm{C}$. Remove and discard trypsin solution; wash in DMEM.

- Incubate in collagenase solution $(1 \mathrm{ml})$ for $30 \mathrm{~min}$ at $37^{\circ} \mathrm{C}$.

- Remove the collagenase digest, discard and replace with fresh solution for $60 \mathrm{~min}$ at $37^{\circ} \mathrm{C}$.

- Keep the final digest and transfer to a 15-ml centrifuge tube. Wash remaining bone fragments with DMEM $(5 \mathrm{ml})$, add the solution to the final digest. 
- Spin at $1500 \mathrm{~g}$ for $5 \mathrm{~min}$. Discard the supernatant and resuspend the cells in DMEM (1 ml). The initial cell suspension may be slightly more heterogeneous compared with a calvarial bone isolate but behaves similarly on culture in osDMEM. ${ }^{4}$

- Add $20 \mathrm{ml}$ of sDMEM to a $75-\mathrm{cm}^{2}$ flasks; add cell suspension (1 ml per flask).

- Incubate the flask at $37^{\circ} \mathrm{C} / 5 \% \mathrm{CO}_{2}$, until the cells become confluent ( $\sim 3-4$ days).

Isolation of primary osteoblasts from human shoulder humeral head trabecular bone

- Patients who are undergoing total shoulder replacements for osteoarthritis can provide a source of bone for explant cultures (consult local Ethical Committee for guidelines on seeking ethical approval and patient consent). This tissue is remote from the shoulder joint and free from contaminating soft tissue. Transfer tissue into a sterile container with $\mathrm{PBS}+\mathrm{AB} / \mathrm{AM}$ and transport to laboratory with minimal delay (Note 7).

- Remove soft connective tissue and coagulated marrow from the outer surfaces of the bone by scraping.
- Place tissue in sterile petri dish containing 5-10 ml of PBS + $A B / A M$. Using rongeurs, bone cutters and scissors carefully cut the bone into 2-4 mm fragments.

- Carefully decant the PBS + AB/AM and transfer the fragments into a 50-ml universal container with 10-15 ml PBS + AB/AM. Vortex for $3 \times 5 \mathrm{~s}$; allow bone fragments to settle for $30 \mathrm{~s}$. Carefully decant off the supernatant to remove haematopoietic marrow, marrow fat and dislodged cells. Add additional $10-15 \mathrm{ml}$ of PBS $+\mathrm{AB} / \mathrm{AM}$ and vortex as before. Repeat this process at least six times or until no remaining haematopoietic marrow and marrow fat is visible and the bone fragments have assumed a white ivory-like appearance (Note 8).

- Incubate the fragments in $1 \%$ trypsin for 10 min at $37^{\circ} \mathrm{C}$. Remove and discard trypsin solution; wash in DMEM, then PBS.

- Incubate in $0.2 \%$ collagenase solution (enough to cover the fragments) for $30 \mathrm{~min}$ at $37^{\circ} \mathrm{C}$.

- Remove the collagenase digest, discard and wash with DMEM.

- Culture these fragments as explants at a density of $0.2-0.5 \mathrm{~g}$ tissue per $100 \mathrm{~mm}$ diameter in a petri dish or a $75-\mathrm{cm}^{3}$ flask in $10 \mathrm{ml}$ of DMEM at $37^{\circ} \mathrm{C} / 5 \% \mathrm{CO}_{2}$.

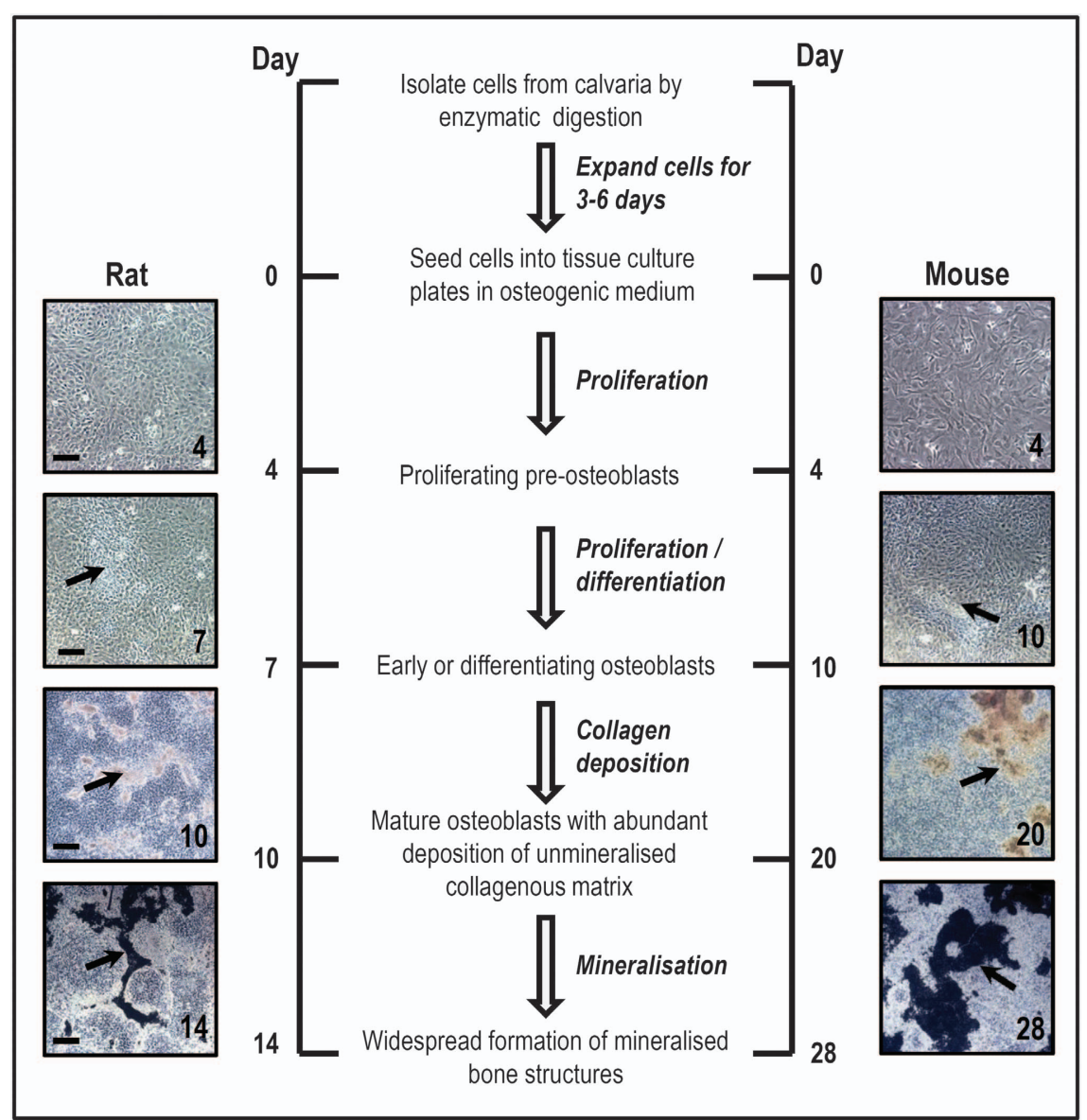

Figure 1 Calvarial osteoblast cultures. Rat calvarial osteoblast cultures are $\sim 14$ days in duration when grown in DMEM. Representative images of unstained cell layers show that by day 4 of culture, a confluent monolayer of preosteoblasts is evident. At day 7, the cells are more compacted and organic matrix is starting to be deposited (as shown by the arrow). By day 10, there is abundant deposition of unmineralised collagenous matrix, and after 14 days there is widespread formation of mineralised bone structures. Mouse osteoblasts take $\sim 28$ days to form mineralised matrix nodules. Representative images of mouse cells show the defined stages of differentiation at $4,10,20$ and 28 days of culture. Scale bars: day $4 / 7=50 \mu \mathrm{m}$, days $10 / 14=500 \mu \mathrm{m}$. 
- Leave the cultures undisturbed for 7 days to ensure the outgrowing cells adhere to the tissue culture plastic. The first cells will be observed after 7-10 days of culture; cells will be confluent after 6-8 weeks. Medium should be exchanged every 7 days.

Osteoblast bone mineralisation assay. This protocol is used for rodent and human osteoblasts.

- Once the cells are confluent remove the media and wash with PBS. Add $1 \mathrm{ml}$ per flask trypsin-EDTA (rodent) or $2 \mathrm{ml}$ per flask Accutase (human) and incubate for $10-15 \mathrm{~min}$ at $37^{\circ} \mathrm{C}$.

- Add $\alpha$ MEM $(10 \mathrm{ml})$ to inactivate the trypsin; transfer the cell suspension to a $15 \mathrm{ml}$ centrifuge tube.

- Spin at $1500 \mathrm{~g}$ for $5 \mathrm{~min}$. Discard the supernatant and resuspend the cells in $\alpha$ MEM or DMEM (1 ml per flask).

- Perform a cell count using a haemocytometer; seed the cells in tissue culture trays (Note 6) in osMEM or osDMEM. Human osteoblasts should be seeded in DMEM to allow cell growth; once confluent, medium can be replaced with osDMEM (Note 3 ). This is day 1 of culture.

- Exchange half the medium every 2-3 days.

- Rodent cultures will be fully confluent by day 4; the overall culture duration will depend on the cells used (Note 9). Rat calvarial osteoblasts will begin to form discreet bone nodules from day 10 with extensive mineralised nodules present by day 14 (Figure 1). Mouse osteoblasts are significantly slower with bone nodules not evident until $\sim 21$ days (Figure 1). Rat long-bone osteoblasts will display collagen deposition from day 10 , giving rise to dense, and organised trabecular-shaped networks by day 20 (Figure 2). Collagen deposition and mineralisation can be visualised in detail by electron microscopy (Figure 3).

- Human osteoblasts from younger patients ( $<40$ years old) will begin to form bone nodules from day 21 giving rise to dense, organised trabecular-shaped networks by day 36 . Human osteoblasts from aged diseased individuals (>65 years old) will form bone nodules from day 36 giving rise to organised trabecular-shaped networks by day 56. It is important to note that depending on disease state and age/sex/race of the patient, time taken for in vitro bone formation may vary (Figure 4 , Note 10).

- The pH should be monitored throughout and maintained at $\sim \mathrm{pH} 7.4$ by adding $6 \mathrm{M} \mathrm{NaOH}$ if required (Note 5).

- TNAP activity, collagen production, cell number and gene expression can be measured at clearly defined stages of osteoblast differentiation (Figures 1, 2 and 5, Note 9).

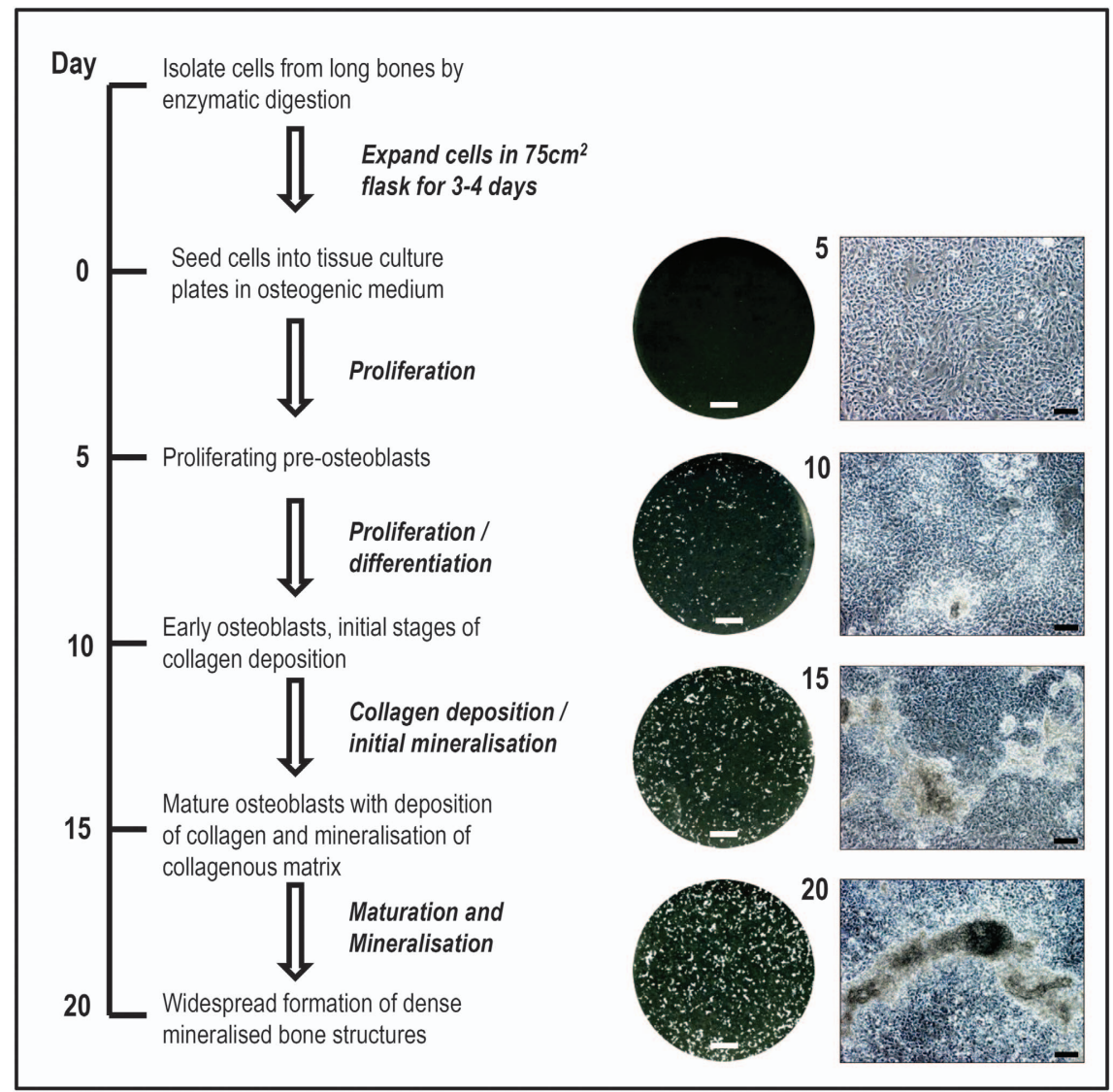

Figure 2 Long-bone osteoblast cultures. Rat long-bone osteoblast cultures are 15-20 days in duration when grown in DMEM. Representative images of unstained cell layers and bone formation show that by day 5 of culture, a confluent monolayer of preosteoblasts is evident. At day 10 , the cells are more compacted and the initial stages of collagen matrix deposition are evident. The collagen matrix is much denser by day 15 and mineralisation of collagenous structures can be seen. By day 20 , there is abundant deposition of mineralised bone structures. Scale bars: whole-well scan $=50 \mathrm{~mm}$, phase contrast images $=100 \mu \mathrm{m}$. 

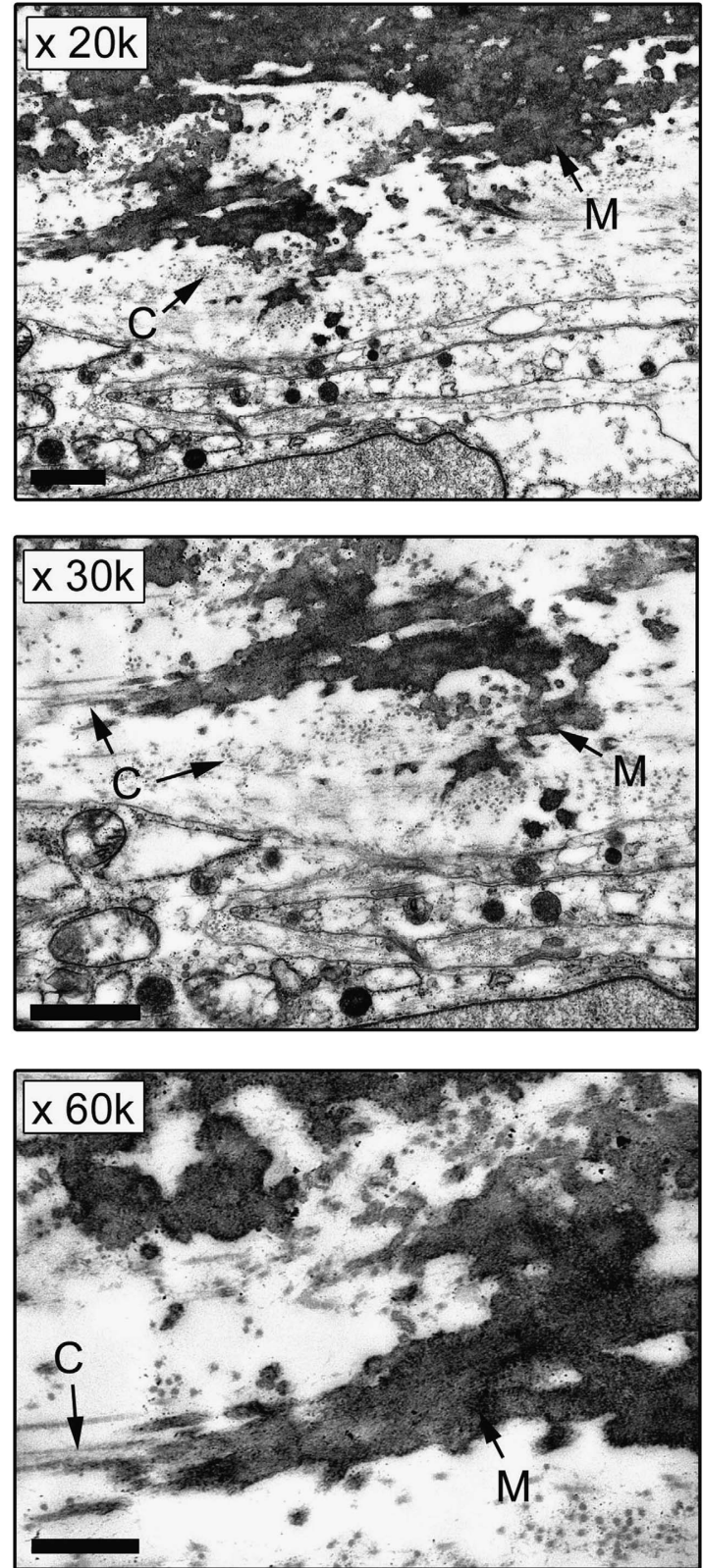

Figure 3 TEM images of the extracellular matrix produced by long-bone osteoblasts. Long-bone osteoblasts were grown in osDMEM for 28 days and then viewed by TEM. $\mathbf{C}=$ collagen fibres, which can be seen longitudinally and in transverse section (where they appear as dots). $\mathbf{M}=$ specific mineralisation of the collagenous extracellular matrix. Magnification at $\times 20 \mathrm{k}$ and $\times 30 \mathrm{k}$ scale bar $=1 \mu \mathrm{m}, \times 60 \mathrm{k}$ scale bar $=500 \mathrm{~nm}$.

\section{Fixation and staining}

- On termination of the experiment, carefully wash the cell layers with PBS. Transfer to appropriate fixative:

- Alizarin red: $2.5 \%$ glutaraldehyde for $5 \mathrm{~min}$

- Sirius red: $70 \%$ ethanol for $1 \mathrm{~h}$

- Masson's trichrome: Bouin's fixative overnight

- TNAP: $4 \%$ paraformaldehyde $(\mathrm{pH} 7.4)$ for 20 min at $4{ }^{\circ} \mathrm{C}$

- Wash twice with PBS and leave to dry.
- Once fully dry, scan unstained plates at 2000 dots $\mathrm{cm}^{2}$ on a high-resolution flat-bed scanner (Note 11) for analysis of mineralised nodule formation.

- Stain as required (Figures 5-8):

- Three times $70 \%$ ethanol washes, leave to dry. Alizarin red for 5 min followed by three times $50 \%$ ethanol washes.

- Sirius red for $1 \mathrm{~h}$; wash with $\mathrm{dH}_{2} \mathrm{O}$ and allow to dry.

- Masson's trichrome collagen stain: Following fixation wash the cell layers with running water until the yellow colour disappears. Stain with Weigert's haematoxylin for $10 \mathrm{~min}$; wash with tap water. Stain with $0.5 \%$ acid fuchsin solution for 5 min and rinse with $\mathrm{dH}_{2} \mathrm{O}$. Treat with phosphomolybdic acid solution for $5 \mathrm{~min}$ before staining with $2 \%$ methyl blue for 5 min. Rinse with $\mathrm{dH}_{2} \mathrm{O}$, followed by $1 \%$ acetic acid for 2 min. Dehydrate the cell layers by several washes with 50, 70 and $100 \%$ alcohol. Nuclei should appear black, cytoplasm red and collagen fibres blue.

- TNAP stain: In glass vials, dissolve $1 \mathrm{mg}$ perml napthol AS-MX-phosphate in dimethylformamide and $1 \mathrm{mg}$ perml of fast blue-BB salt in $0.1 \mathrm{M}$ Tris $\mathrm{HCl} \mathrm{pH} \mathrm{9.2.} \mathrm{Combine}$ both solutions and mix well; filter through a $0.45 \mu \mathrm{m}$ syringe filter. Cover cells evenly $(0.2 \mathrm{ml}$ per well in a 12 -well tray) and incubate the plates for $25 \mathrm{~min}$ at $37^{\circ} \mathrm{C}$ in the dark. Remove stain solution and wash the monolayers twice in $\mathrm{dH}_{2} \mathrm{O}$.

\section{Quantitation of mineralised nodule formation}

- Several programs are available, such as Adobe Photoshop and Image J (http://rsbweb.nih.gov/ij/), which can be used to determine the number and surface area of mineralised bone nodules.

- Using these programs, binary images of each individual well can be subjected to automated analysis, using constant threshold and minimum particle levels (Figure 9).

\section{Notes}

1. Tissue culture medium. For successful osteoblast cultures it is important to use the correct tissue culture medium. Mouse osteoblasts appear to require more nutritional support in vitro and to get significant mineralisation should be grown in $\alpha M E M .^{12}$ The formulation of $\alpha M E M$ is designed to closely approximate the protein composition of cells ${ }^{13}$ and it contains higher concentrations of amino acids and nucleotides compared with other tissue culture mediums (such as DMEM). Rat osteoblasts will successfully mineralise in both $\alpha \mathrm{MEM}$ and DMEM; however, cultures will be 2-3 days shorter when grown in the former. The timelines in Figures $\mathbf{1}$ and $\mathbf{2}$ are based on mouse and rat osteoblasts cultured in osMEM and osDMEM, respectively.

2. $\beta$-Glycerophosphate. Bone mineralisation requires a constant supply of $\mathrm{Ca}^{2+}$ and orthophosphate $\left(\mathrm{P}_{\mathrm{i}}\right)$. The main source of $\mathrm{Pi}$ in bone is TNAP, which hydrolyses a range of phosphatecontaining substrates including ATP, pyrophosphate and $\beta$-glycerophosphate. To form mineralised bone nodules in vitro an exogenous source of phosphate is required; most commonly used is $\beta$-glycerophosphate $(2-10 \mathrm{~mm})$. Previous studies have 


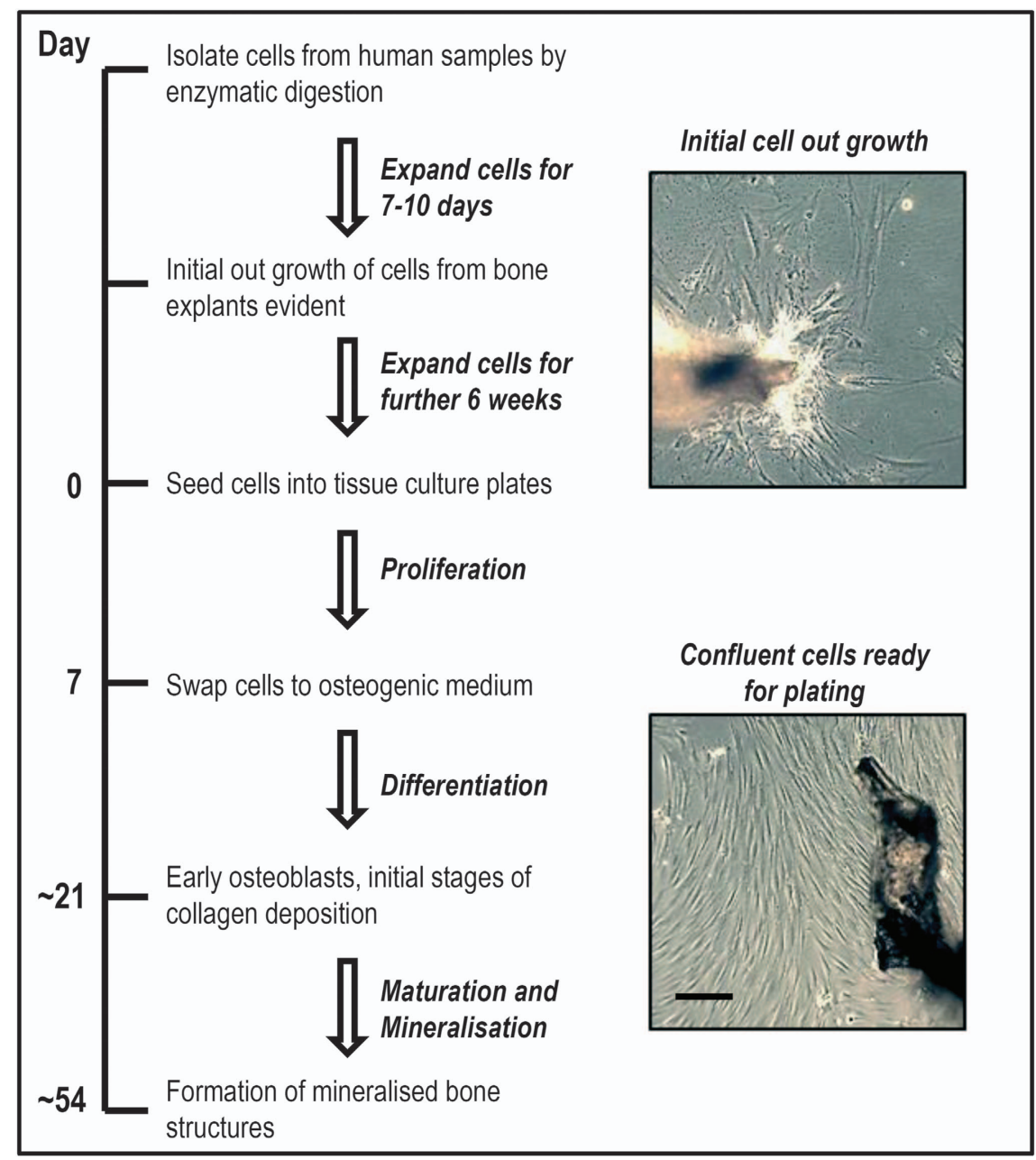

Figure 4 Human humeral head trabecular bone explant cultures. Human osteoblasts take $\sim 6-8$ weeks to reach subconfluence when cultured in DMEM by the explant method. Representative images show unstained cell layers from explant bone at initial out growth and confluent monolayers of human humeral head trabecular osteoblasts before subculture. Following subculture mineralised bone structures are usually evident after $\sim 54$ days. Scale bar $=50 \mu \mathrm{m}$.

shown in cultures of rat osteoblasts, $2 \mathrm{~mm} \beta$-glycerophosphate reproducibly forms abundant, discrete nodules with a characteristic 'trabecular-shaped' morphology ${ }^{1,5}$ (Figure 6). However, culture with 5-10 mm $\beta$-glycerophosphate reduces cell viability and causes widespread, non-specific mineral deposition that differs from true-bone formation ${ }^{1,5}$ (Figure 9). Mouse osteoblasts can tolerate a higher concentration of $\beta$-glycerophosphate (up to $5 \mathrm{~mm}$ ) before non-specific mineralisation occurs. ${ }^{12}$ Thus, when investigating osteoblast function it is critical that the appropriate, low concentration of $\beta$-glycerophosphate is used. This ensures that any effects on bone formation/mineralisation are due to differences in osteoblast activity rather than an excess level of $\mathrm{P}_{\mathrm{i}}$ in the culture medium.

3. Dexamethasone. The ability of dexamethasone to promote osteoblast differentiation in rat cells was first identified in the $1980 \mathrm{~s}^{14}$ and it is now routinely added to these cultures in the nanomolar range. ${ }^{1}$ In contrast, many studies have shown that dexamethasone inhibits osteogenic differentiation in murine cells. ${ }^{12,15-19}$ Using the above protocols, we have recently
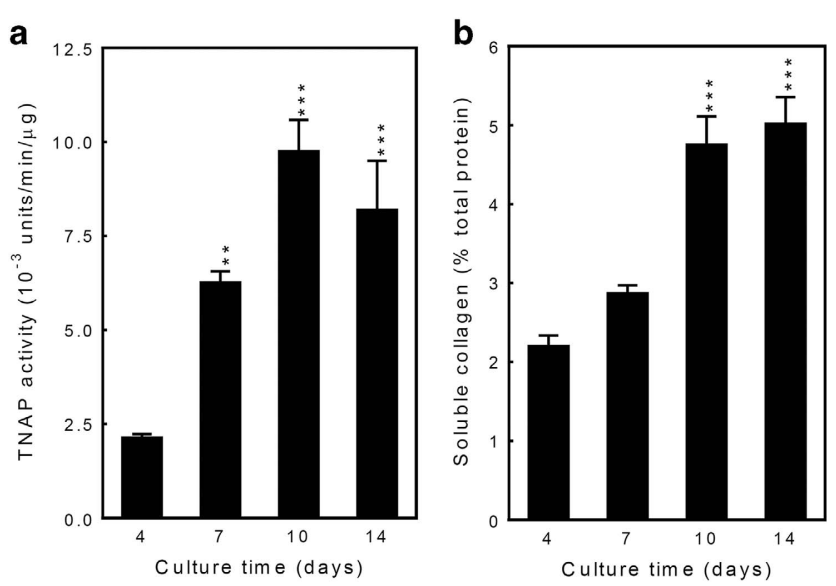

Figure 5 TNAP activity and soluble collagen levels in rat osteoblast cultures. TNAP activity and soluble collagen levels were measured at regular intervals throughout the osteoblast culture period. (a) TNAP activity was 2.5 -fold and $\leqslant$ fourfold higher in differentiating and mature osteoblasts, respectively, compared with proliferating, preosteoblasts. (b) Soluble collagen levels were increased up to twofold in mature osteoblasts. Values are mean \pm s.e.m.; ${ }^{* *} P<0.01,{ }^{* * *} P<0.001$. 
a

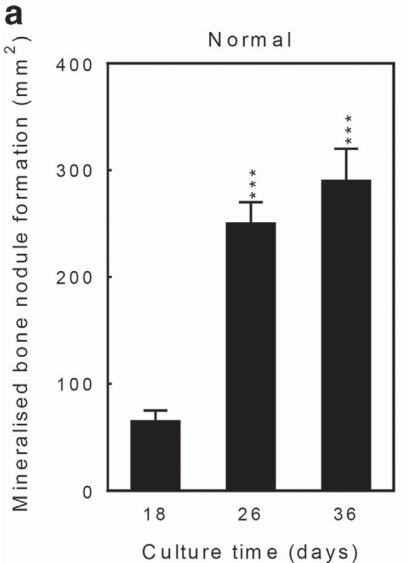

C

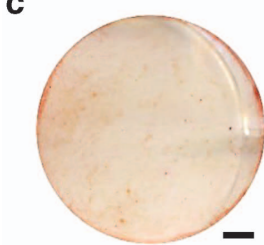

Day 18

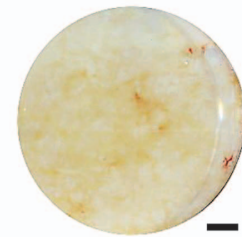

Day

36 b

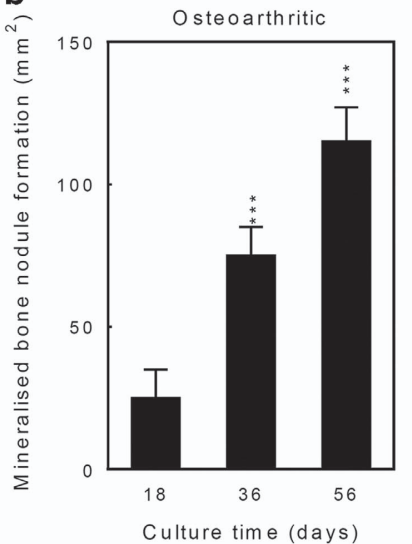

Culture time (days) d

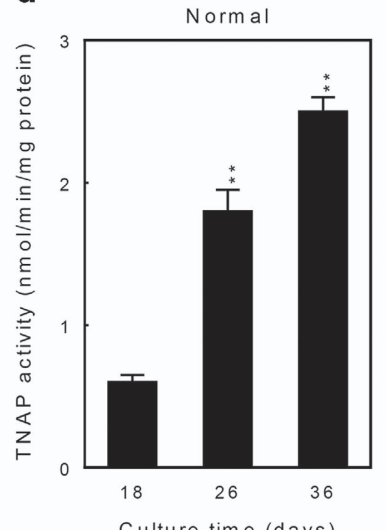

Culture time (days)

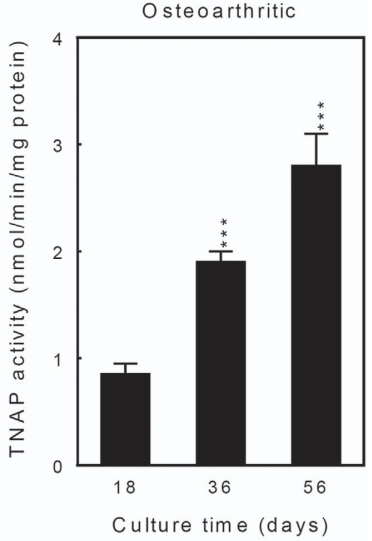

f
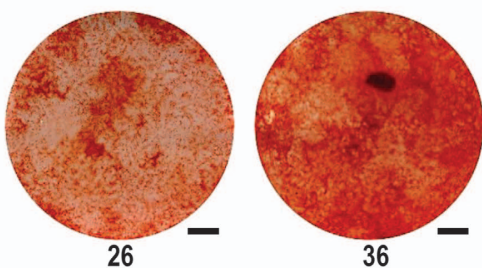

Normal

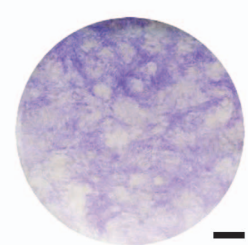

18
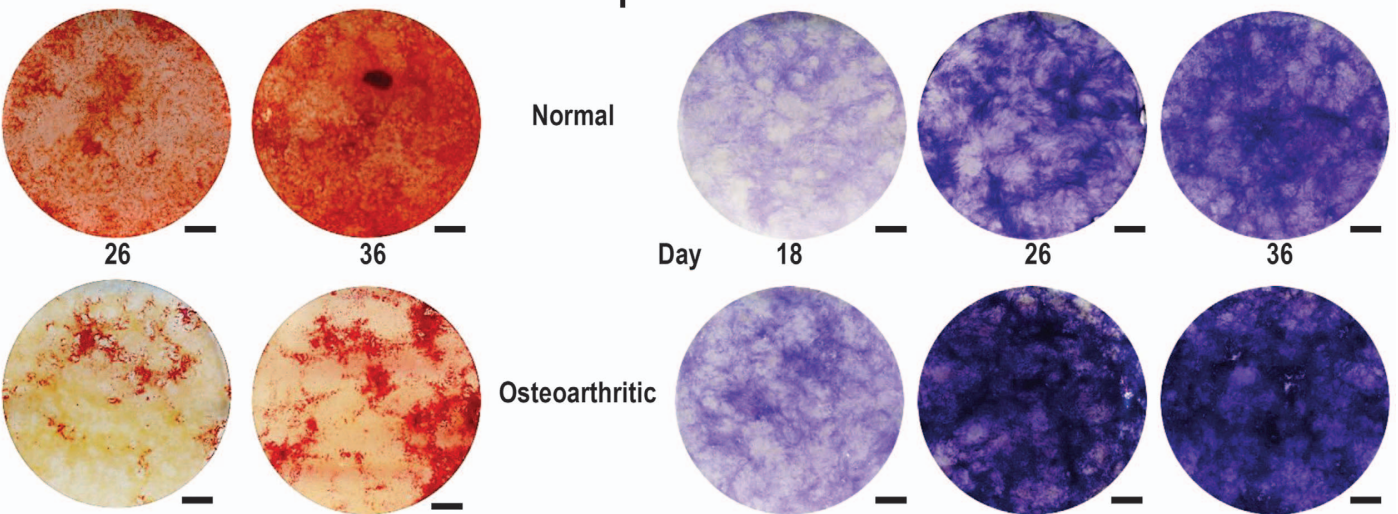

56

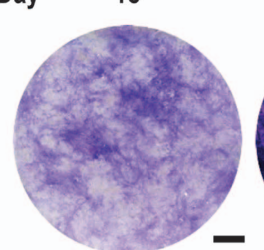

Day

18

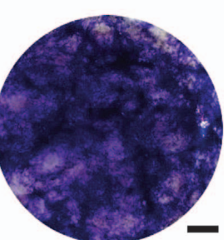

36

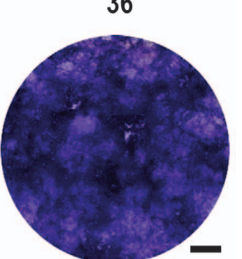

56

Figure 6 TNAP activity and mineralisation in human trabecular osteoblasts from normal and osteoarthritic patients. The level of mineralised nodule formation increases with culture time in human osteoblasts from (a) normal (30 years old) and (b) osteoarthritic patients ( 86 years old). (c) Representative whole-well scans of alizarin red stained human osteoblasts at different stages of culture. Human cells appear to be unable to form the discrete trabecular bone structures characteristic of neonatal rodent osteoblasts; instead, the mineralisation is more widespread. Human osteoblasts from (d) normal and (e) osteoarthritic patients display TNAP activity throughout the culture period. (f) Representative images showing increased levels of TNAP staining with osteoblast differentiation. Values are mean \pm s.e.m.; ${ }^{* *} P<0.01,{ }^{* *} P<0.001$. Scale bar $=50 \mathrm{~mm}$.

shown that $10 \mathrm{~nm}$ dexamethasone is required for differentiation in rat osteoblast cultures but slows differentiation in mouse cells. $^{12}$ Thus, we recommend that dexamethasone is only added to osteogenic medium when growing rat cells.

Because of the slow growth of human cells, dexamethasone should only be added from confluence; however, ascorbate can be added to facilitate cell growth.

4. The importance of $\mathrm{pH}$. Extracellular $\mathrm{pH}$ is an important factor in the regulation of bone mineralisation. ${ }^{6}$ Acidosis inhibits matrix mineralisation by reducing TNAP expression and activity, while increasing mineral solubility. ${ }^{6}$ The metabolic activity of osteoblasts will cause progressive medium acidification especially in mature, bone-forming cultures when cell numbers are high. To ensure that $\mathrm{pH}$ does not influence mineralisation, it may be required to add $\mathrm{OH}^{-}$ions (as $6 \mathrm{M} \mathrm{NaOH}$ ) to the culture medium to ensure a $\mathrm{pH}$ of $\sim 7.4$.

5. Accutase. This is a gentler alternative to trypsin-EDTA for cell dissociation. It reduces the level of nonviable cells and does not require serum inhibition.
6. Osteoblast seeding density and tissue culture plates. Rat and human osteoblasts can be cultured in several different well plate formats. Typical seeding densities per well are $2.5 \times 10^{4}$ (24-well), $5 \times 10^{4}$ (12-well) and $10^{5}$ (6-well). Mouse osteoblasts will only form abundant mineralised bone nodules in 6-well trays $\left(10^{5}\right.$ cells per well). Seeding cells in 24 - or 12 -well trays will result in significant monolayer peeling before mineralisation. ${ }^{12}$

7. Human tissue storage and transport. To avoid degradation of viable cells it is important to place the bone samples in explants as soon as possible. Store specimens before explant for not $>24 \mathrm{~h}$ at $4{ }^{\circ} \mathrm{C}$ in PBS ( + AB/AM to the risk of reduce post-operative culture contamination) or serum-free DMEM.

8. Removal of marrow and fat from human bone. Samples from diseased and aged bone can contain high amounts of marrow and fat. This must be removed to avoid any adipocyte or stromal cell contamination.

9. Stages of differentiation in osteoblast cultures. These methods allow rodent osteoblasts to be studied at clearly identified stages of differentiation such as proliferating pre-osteoblasts, 


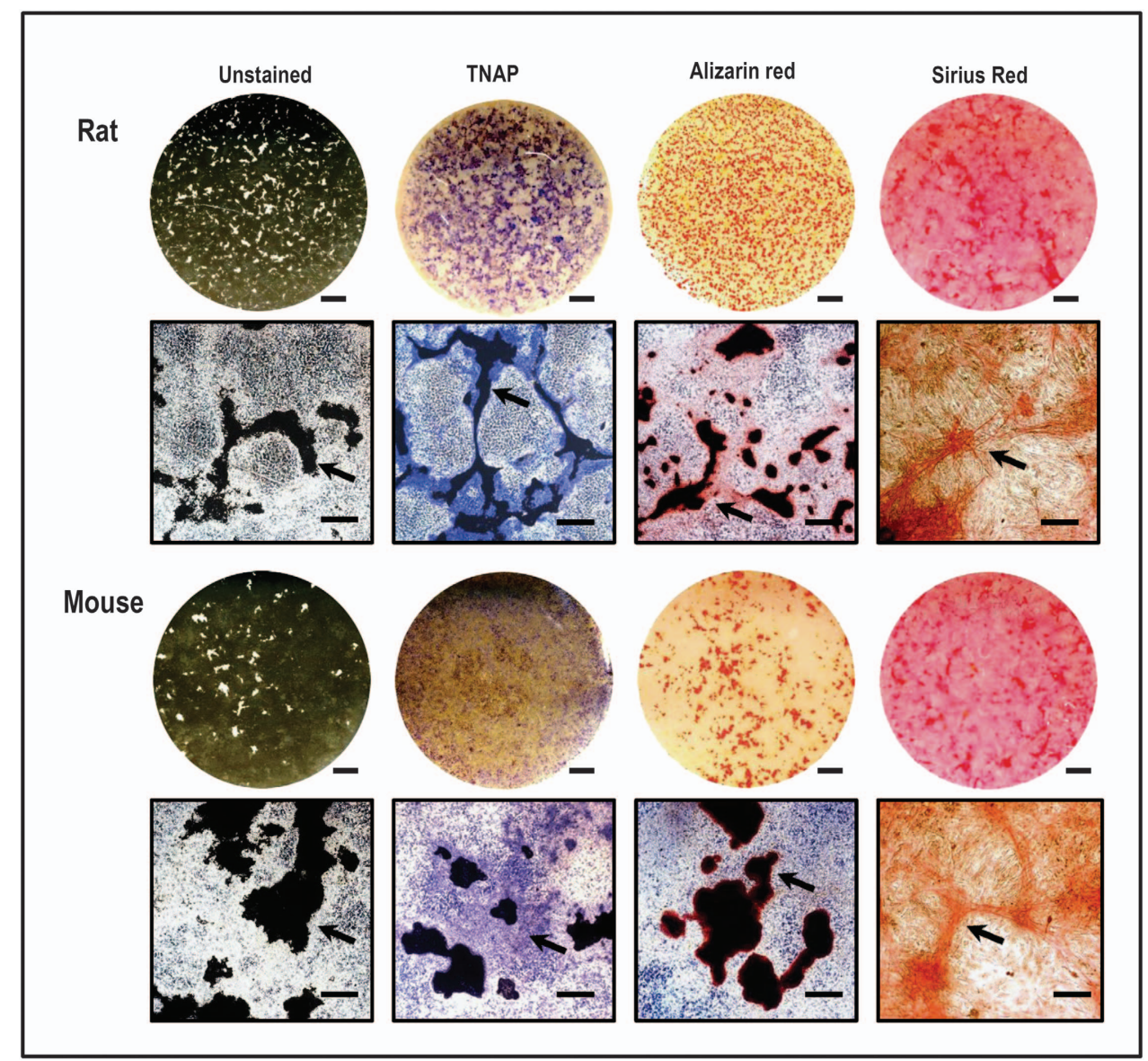

Figure 7 Different staining methods in primary calvarial osteoblast cultures. Images show 14-day rat and 28-day mouse osteoblast cultures either left unstained, or stained with TNAP, alizarin red or sirius red. The individual bone structures formed by rodent osteoblasts displayed marked differences in appearance. Rat osteoblasts typically produced smaller bone nodules that had a 'trabecular-shaped' appearance, whereas in mouse osteoblast cultures the bone structures were larger but fewer in numbers. TNAP staining was closely associated with the mineralised matrix in rat osteoblast cultures but more widespread in mouse osteoblasts. Sirius red staining shows the presence of collagen fibres in these cultures. Scale bars $=50 \mathrm{~mm}$ (whole-well scans) and $500 \mu \mathrm{m}$ (phase contrast microscopy images).

early or differentiating osteoblasts, mature osteoblasts and mature, mineralising osteoblasts (Figures 1, 2 and 5).

10. Young vs old cells. Human osteoblasts (particularly from older individuals) tend to grow and differentiate slower compared with neonatal rodent osteoblasts. Cells from young animals have considerable growth potential and thus will expand rapidly in vitro. In contrast, cells from adult animals (including human donors) may have progressed too far towards their 'Hayflick limit' (the number of times a cell population will divide until cell division is arrested ${ }^{20}$ ) to be capable of rapid expansion and differentiation.

11. Analysis of mineralised nodule formation. A commonly used method for quantifying bone formation is image analysis of alizarin red stained osteoblast cell layers. However, this technique has limitations and, if staining is performed incorrectly, can give inaccurate results. For example, when cell layers are not stained sufficiently the alizarin red will not reach the centre of the bone nodules leaving an unstained white region. Conversely, if the cells are not washed properly following staining the alizarin red can 'bleed' into the region immediately surrounding the bone structure. Figure 9a shows the same cultures before and after alizarin red staining. The corresponding binary images shown in Figure $9 \mathrm{~b}$ highlight the obvious differences in the level of bone mineral detected. Comparison of stained and unstained plates by automated analysis shows that the level of bone formation is $\leqslant$ fourfold higher in the stained plates (Figure 9c). Performing analysis of bone formation on unstained plates removes this imprecision and provides a more reliable representation of the actual level of mineralisation. Culture with excess $\beta$-glycerophosphate (10 mm) amplifies this problem due to the widespread nonspecific deposition of mineral (Figure 9).

Bone formation can also be quantified by eluting the alizarin red with acidic solutions and measuring the optical density by spectrophotometry. This process will dissolve all the stain into solution, whether it is associated with a discrete bone nodule or is nonspecific staining, and therefore can also give inaccurate results.

\section{Discussion}

The methods described here provide a robust in vitro model for studying the regulation of osteoblast proliferation, differentiation and function. However, these protocols do have some 


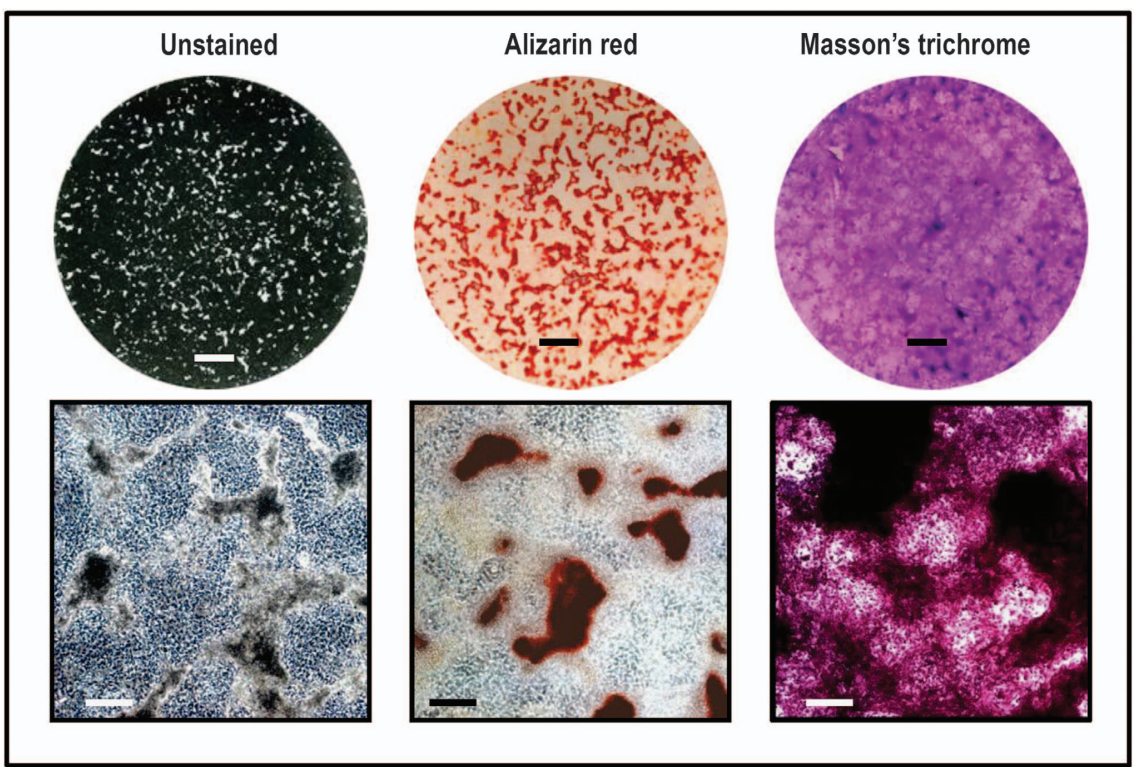

Figure 8 Different staining methods in primary long-bone osteoblast cultures. Images show 20-day rat long-bone osteoblast cultures either left unstained, or stained with alizarin red or Masson's trichrome. The rat long-bone osteoblasts produce 'trabecular-shaped' bony structures that display positive staining for calcium and collagen deposition. Scale bars $=50 \mathrm{~mm}$ (whole-well scans) and $500 \mu \mathrm{m}$ (phase contrast microscopy images).

a

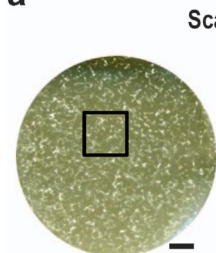

$2 \mathrm{mM} \beta$-GP
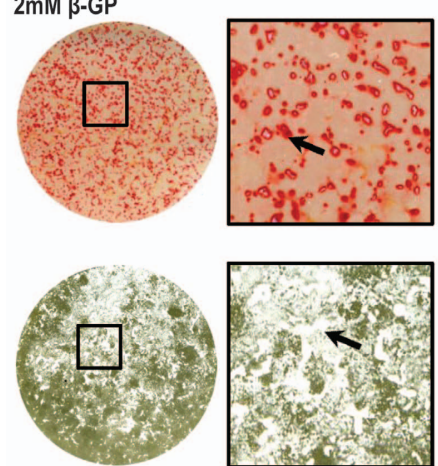

$10 \mathrm{mM} \beta-\mathrm{GP}$
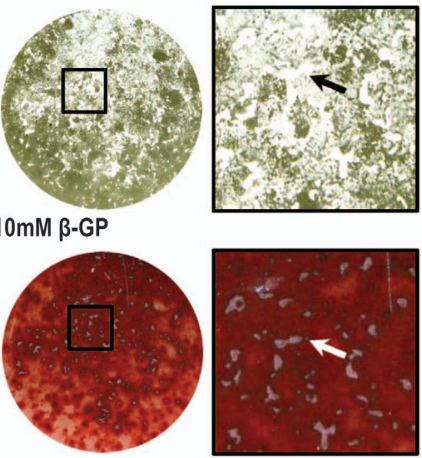

b
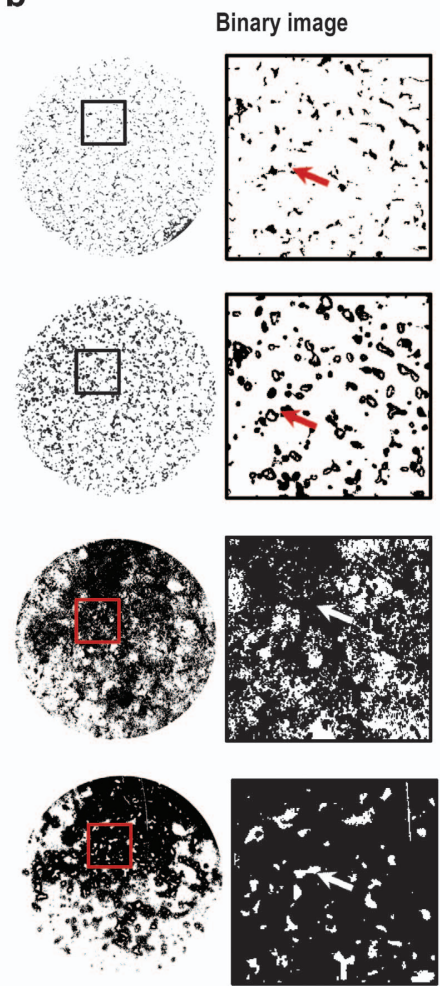

C

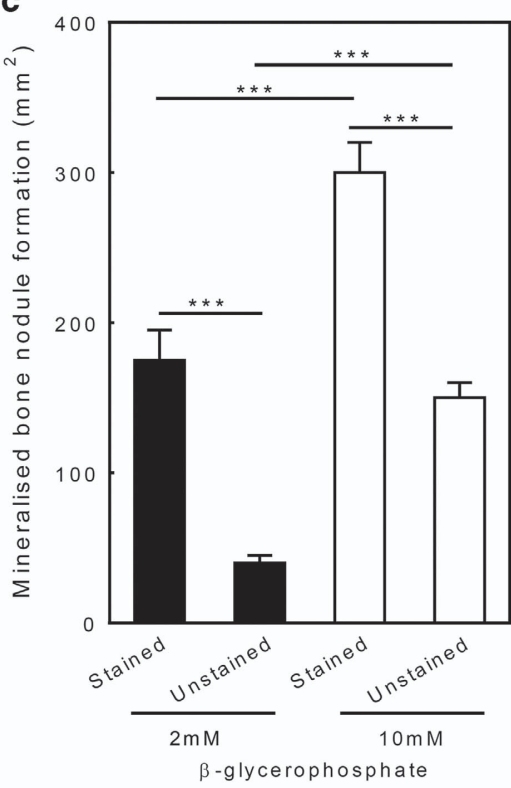

Figure 9 Quantification of bone mineralisation by image analysis. The representative images in (a) are of the same cell layers before and after alizarin red staining; the stained images are examples of cultures that have been inadequately washed, leading to bleeding of the alizarin red into the surrounding cells (shown by the arrows). (b) Binary images of the same cell layers highlight the differences in the level of bone mineral detected between stained and unstained plates. (c) Comparison of stained and unstained plates by automated analysis shows that the level of bone formation is $\leqslant$ fourfold higher in the stained plates. Culture with excess $\beta$-glycerophosphate ( $10 \mathrm{~mm})$ amplifies this problem due to the widespread nonspecific deposition of mineral. Scale bars $=50 \mathrm{~mm}$ (whole-well scans) and $5 \mathrm{~mm}$ (nodule images). Values are mean \pm s.e.m.; ${ }^{* * *} P<0.001$.

limitations that need to be taken into account when planning and interpreting experiments. First, the use of high levels of $\beta$ glycerophosphate will result in nonspecific, dystrophic mineralisation, which is not true-bone formation. Second, as the osteoblasts are grown in monolayers they cannot form the three-dimensional (3D) cell-to-cell interactions, which occur 
in vivo. Third, although rodent osteoblasts are readily available, the supply of human osteoblasts is limited and often restricted to cells from older patients undergoing surgery. Finally, once in culture, human osteoblasts are often very slow growing and therefore it takes a significant amount of time to obtain experimental data.

There are some newer approaches/techniques that can be used to address some of these issues. The development of 3D culture systems has provided a novel method for growing osteoblasts (in isolation or cocultured with other cell types) in an environment that more accurately represents the in vivo situation. ${ }^{21,22}$ There have also been several studies that have described the isolation of human osteoblast precursors from peripheral or cord blood. ${ }^{23-25}$ At present, work using these methods is limited; however, they do represent a potentially important source of human osteoblasts.

\section{Recommended Future Reading}

There are many other papers that cover different methods for isolating and culturing osteoblasts. The following publications are recommended for further information about rodent and human osteoblasts:

Orriss IR, Hajjawi MOR, Huesa C, MacRae VE, Arnett TR. Optimisation of the differing conditions required for bone formation in vitro by primary osteoblasts from mice and rats. Int J Mol Med 2014;34:1201-1208.

Dillon JP, Waring-Green VJ, Taylor AM, Wilson PJ, Birch M, Gartland A et al. Primary human osteoblast cultures. Methods Mol Biol 2012;816:3-18.

Detailed methodological information about the 3D coculture of osteoblasts with other cell types is provided by:

Unger RE, Halstenberg S, Sartoris A, Kirkpatrick CJ. Human endothelial and osteoblast cocultures on 3D biomaterials. Methods Mol Biol 2011;695:229-241.

\section{Acknowledgements}

We are grateful for funding from Arthritis Research, UK and Orthopaedic Research, UK. We thank Professor Tim Arnett, University College London, for his support and expertise. We also acknowledge Professor Adrienne Flanagan, Mr Rob Pollock, Professor Roger Emery and surgical teams at the Royal National Orthopaedic Hospital and Imperial NHS trusts. Supported by the IBMS-ECTS Young Investigators.

\section{Conflict of Interest}

The authors declare no conflict of interest.

\section{References}

1. Orriss IR, Taylor SE, Arnett TR. Rat osteoblast cultures. Methods Mol Biol 2012;816:31-41.

2. Dillon JP, Waring-Green VJ, Taylor AM, Wilson PJ, Birch M, Gartland A et al. Primary human osteoblast cultures. Methods Mol Biol 2012;816:3-18.

3. Bakker AD, Klein-Nulend J. Osteoblast isolation from murine calvaria and long bones. Methods Mol Biol 2012;816:19-29.

4. Taylor SEB, Key ML, Lander M, Orriss IR, Patel JJ, Arnett TR. A novel method for the isolation and culture of rat long bone osteoblasts. Bone 2009;44:S318-S319.

5. Orriss IR, Utting JC, Brandao-Burch A, Colston K, Grubb BR, Burnstock G et al. Extracellular nucleotides block bone mineralization in vitro: evidence for dual inhibitory mechanisms involving both $\mathrm{P}_{2} \mathrm{Y}_{2}$ receptors and pyrophosphate. Endocrinology 2007; 148:4208-4216

6. Brandao-Burch A, Utting JC, Orriss IR, Arnett TR. Acidosis inhibits bone formation by osteoblasts in vitro by preventing mineralization. Calcif Tissue Int 2005;77:167-174.

7. Utting JC, Robins SP, Brandao-Burch A, Orriss IR, Behar J, Arnett TR. Hypoxia inhibits the growth, differentiation and bone-forming capacity of rat osteoblasts. Exp Cell Res 2006;312:1693-1702.

8. Siggelkow H, Rebenstorff K, Kurre W, Niedhart C, Engel I, Schulz H et al. Development of the osteoblast phenotype in primary human osteoblasts in culture: comparison with rat calvarial cells in osteoblast differentiation. J Cell Biochem 1999;75:22-35.

9. Beresford JN, Graves SE, Smoothy CA. Formation of mineralized nodules by bone derived cells in vitro: a model of bone formation? Am J Med Genet 1993;45:163-178.

10. Beresford JN, Gallagher JA, Poser JW, Russell RG. Production of osteocalcin by human bone cells in vitro. Effects of $1,25(\mathrm{OH}) 2 \mathrm{D} 3,24,25(\mathrm{OH}) 2 \mathrm{D} 3$, parathyroid hormone, and glucocorticoids. Metab Bone Dis Relat Res 1984;5:229-234.

11. Couchourel D, Aubry I, Delalandre A, Lavigne M, Martel-Pelletier J, Pelletier JP et al. Altered mineralization of human osteoarthritic osteoblasts is attributable to abnormal type I collagen production. Arthritis Rheum 2009:60:1438-1450.

12. Orriss IR, Hajjawi MOR, Huesa C, MacRae VE, Arnett TR. Optimisation of the differing conditions required for bone formation in vitro by primary osteoblasts from mice and rats. Int $\mathrm{J}$ Mol Med 2014:34:1201-1208.

13. Eagle H. Nutrition needs of mammalian cells in tissue culture. Science 1955;122:501-514.

14. Bellows CG, Aubin JE, Heersche JN, Antosz ME. Mineralized bone nodules formed in vitro from enzymatically released rat calvaria cell populations. Calcif Tissue Int 1986;38: 143-154.

15. Chen TL, Cone CM, Feldman D. Glucocorticoid modulation of cell proliferation in cultured osteoblast-like bone cells: differences between rat and mouse. Endocrinology 1983;112: $1739-1745$.

16. Banerjee C, McCabe LR, Choi JY, Hiebert SW, Stein JL, Stein GS et al. Runt homology domain proteins in osteoblast differentiation: AML3/CBFA1 is a major component of a bone-specific complex. J Cell Biochem 1997;66:1-8.

17. Li H, Qian W, Weng X, Wu Z, Li H, Zhuang Q et al. Glucocorticoid receptor and sequential P53 activation by dexamethasone mediates apoptosis and cell cycle arrest of osteoblastic MC3T3E1 cells. PLOS ONE 2012;7:e37030.

18. Li J, Zhang N, Huang X, Xu J, Fernandes JC, Dai K et al. Dexamethasone shifts bone marrow stromal cells from osteoblasts to adipocytes by C/EBPalpha promoter methylation. Cell Death Dis 2013;4:e832.

19. Esmail MY, Sun L, Yu L, Xu H, Shi L, Zhang J. Effects of PEMF and glucocorticoids on proliferation and differentiation of osteoblasts. Electromagn Biol Med 2012;31:375-381.

20. Hayflick L. The limited in vitro lifetime of human diploid cell strains. Exp Cell Res 1965;37: 614-636.

21. Unger RE, Halstenberg S, Sartoris A, Kirkpatrick CJ. Human endothelial and osteoblast cocultures on 3D biomaterials. Methods Mol Biol 2011;695:229-241.

22. Jones GL, Walton R, Czernuszka J, Griffiths SL, El Haj AJ, Cartmell SH. Primary human osteoblast culture on 3D porous collagen-hydroxyapatite scaffolds. J Biomed Mater Res A 2010:94:1244-1250

23. Eghbali-Fatourechi GZ, Lamsam J, Fraser D, Nagel D, Riggs BL, Khosla S. Circulating osteoblast-lineage cells in humans. N Engl J Med 2005;352:1959-1966.

24. Rosada C, Justesen J, Melsvik D, Ebbesen P, Kassem M. The human umbilical cord blood: a potential source for osteoblast progenitor cells. Calcif Tissue Int 2003;72:135-142.

25. Liu G, Li Y, Sun J, Zhou H, Zhang W, Cui L et al. In vitro and in vivo evaluation of osteogenesis of human umbilical cord blood-derived mesenchymal stem cells on partially demineralized bone matrix. Tissue Eng Part A 2010;16:971-982. 